\title{
La chirurgie non prothétique de la coxarthrose
}

\section{Expérience du GECO}

\author{
R. Petit \\ 16, avenue Clemenceau, F-68100 Mulhouse
}

Ses très brillants résultats font que l'arthroplastie par prothèse totale de hanche s'est imposée dans tous les domaines de la chirurgie de la hanche au cours des vingt dernières années. $\mathrm{Ce}$ n'est pourtant pas par défaut d'une résistance organisée que cette « solution de facilité » a pris le pas sur de nombreuses techniques de traitement non prothétique de la coxarthrose établie.

De nombreuses publications, de brillantes conférences, de savants calculs vectoriels, qui tous nous ont beaucoup appris sur la physiologie et la biomécanique de la hanche, nous ont été présentés. Toute cette science nous a tourmentés pendant deux décennies et nous a culpabilisés devant des indications d'arthroplastie par prothèse chez des patients jugés trop jeunes, ou bien devant des lésions jugées encore accessibles au traitement non prothétique. Le sacrifice osseux délibéré nous a souvent fait reculer au lendemain d'un congrès dont nous restait le souvenir du succès de la réflexion biomécanique dix ans après l'intervention conservatrice.

Pendant ces vingt ans nous avons régulièrement vu des patients porteurs de dysplasies sévères arriver à nos consultations avec des lésions arthrosiques désormais inaccessibles à la belle varisation ou à l'intervention simple de couverture de la tête fémorale, malgré la diffusion large de ces indications salvatrices dans les «médias» médicaux.

Pendant ces vingt ans, la technique des ostéotomies a considérablement progressé, permettant d'éliminer pratiquement la pseudarthrose, et de réaliser des corrections complexes. L'on constate malgré tout un recul des indications d'ostéotomies, la quasi-disparition des arthrodèses et la disparition des résections, avec ou sans angulation sous-trochantérienne, au profit des arthroplasties.

Cette évolution va de pair avec les légitimes exigences de nos patients qui n'acceptent plus vraiment l'essai de l'intervention conservatrice avec ses longs mois de réadaptation, d'absentéisme professionnel, et ses résultats parfois modestes sur le plan fonctionnel.

Nous avons décidé d'entreprendre l'étude rétrospective des interventions non prothétiques effectuées exclusivement sur des lésions arthrosiques confirmées, dans l'espoir de redéfinir des indications raisonnables à ces différentes techniques qui conservent toutes un très grand intérêt. Notre objectif thérapeutique est peut-être exigeant, mais nous pensons interprêter les aspirations de nos patients :

- en considérant par exemple comme mauvais résultat:

- toute reprise avant la cinquième année post-opératoire,
- toute absence d'amélioration de la cotation de la douleur lorsque la cotation pré-opératoire selon Merle d'Aubigné [1] est égale ou inférieure à 4 ,

- toute perte de cotation de la marche/ stabilité lorsque la cotation pré-opératoire est égale ou inférieure à 4 ,

- et en considérant comme bons résultats tous les patients bénéficiant $a$ cinq ans d'une cotation de hanche correspondant aux critères suivants :

- douleur: 5 ou 6,

- mobilité : 4 ou 5 ou 6,

- marche/stabilité : 5 ou 6 .

Après le rappel des considérations biomécaniques, nous présentons les opérés de chacune des techniques sur des bases purement thérapeutiques. L'étude des reprises chirurgicales effectuées chez ces patients est exposée avant de comparer les résultats obtenus par nos ostéotomies aux résultats d'arthroplasties par prothèses sans ciment présentés lors de la réunion du GECO de janvier 1989. Nous essaierons sur ces bases de préciser les indications que nous croyons raisonnables.

\section{Bibliographie}

1. Merle d'Aubigné R (1970) Cotation chiffrée de la fonction de la hanche. Rev Chir Orthop $56: 481-486$ 